\title{
Bilateral Diffuse Uveal Melanocytic Proliferation in a Woman with Metastatic Scalp Squamous Cell Carcinoma Treated with Cemiplimab
}

\author{
Etti Katzburga ${ }^{a}$ Dinah Zur ${ }^{a} \quad$ Orit Gutfeld $^{b}$ Ilya Kirgner ${ }^{c}$ \\ Zohar Habot-Wilner ${ }^{a}$ \\ aDivision of Ophthalmology, Tel Aviv Medical Center, Sackler Faculty of Medicine, Tel \\ Aviv University, Tel Aviv, Israel; b'Division of Oncology, Radiotherapy Institute, Tel Aviv \\ Medical Center, Sackler Faculty of Medicine, Tel Aviv University, Tel Aviv, Israel; 'Division of \\ Hematology, Tel Aviv Medical Center, Sackler Faculty of Medicine, Tel Aviv University, \\ Tel Aviv, Israel
}

\section{Keywords}

Bilateral diffuse uveal melanocytic proliferation - Anti-PD-1 - Cemiplimab - Plasmapheresis .

Scalp squamous cell carcinoma

\begin{abstract}
Bilateral diffuse uveal melanocytic proliferation (BDUMP) is a rare paraneoplastic intraocular syndrome that causes progressive visual loss in patients driven by an IgG factor associated with an underlying malignancy. The IgG factor - cultured melanocyte elongation and proliferation - was found in the IgG fraction of the serum of BDUMP patients. It has been shown to be involved in melanocytic proliferation. In this case report, we describe the first case of BDUMP related to metastatic cutaneous squamous cell carcinoma (CSCC) of the scalp. A 61-yearold woman complained of decreased vision in both of her eyes, while being treated with cemiplimab (an anti-PD-1 therapy) for metastatic cSCC. Fundus examination showed hypopigmented lesions in a leopard pattern and pigmentary clumps in both eyes. Further imaging confirmed the diagnosis of BDUMP. The patient was successfully treated with plasmapheresis. During follow-up, cataract progressed in both eyes, and she underwent cataract surgery with visual acuity improvement to 20/20. BDUMP is a challenging diagnosis especially in patients treated with anti-PD-1 immunotherapy as it can be confused with drug-related effects. It is
\end{abstract}

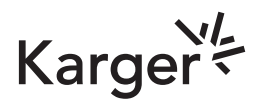


crucial to distinguish between the cases in order to allow the appropriate treatment which includes continuation of systemic anti-PD-1 for the underlying malignancy and plasmapheresis therapy for BDUMP.

(C) 2021 The Author(s).

Published by S. Karger AG, Basel

\section{Introduction}

Bilateral diffuse uveal melanocytic proliferation (BDUMP) is a rare paraneoplastic intraocular syndrome, with around 60 cases reported [1,2]. As first described by Gass et al. [3] in 1990, BDUMP is characterized by multiple, elevated, pigmented uveal lesions, diffuse thickening of the uveal tract, and rapidly progressive cataract. The mechanism of uveal and dermal melanocytic proliferation in BDUMP is not clear. However, a cultured melanocyte elongation and proliferation factor from the IgG fraction of the serum of BDUMP patients has been shown to be involved in melanocytic proliferation [4].

BDUMP is mainly associated with primary endocrine carcinomas; female urogenital (69\% of all female cases) and male lung carcinomas (52\% of all male cases) are the most common related carcinomas. Other sporadic-related carcinomas reported to date include carcinomas of the colon, urinary bladder, pancreatic, esophageal, breast, hepatocellular, Bartholin gland, gallbladder, and renal cell, as well as central nervous system lymphoma [1]. We describe the first case of BDUMP related to metastatic squamous cell carcinoma (SCC) of the scalp in a patient treated with cemiplimab, an anti-programmed cell death protein 1 (PD-1) checkpoint inhibitor.

\section{Case Report}

A 61-year-old woman with a known metastatic cSCC of the scalp was referred to the ophthalmology clinic due to decreased vision in both eyes for 3 months. Eighteen months earlier, the patient was diagnosed with skin SCC in her right occipital scalp. She underwent wide local excision along with neck dissection; however, shortly after surgery, her disease progressed with new nodal and lung metastases. Systemic chemotherapy (cisplatin and 5 -fluorouracil) was commenced, followed by cetuximab, but her disease continued to rapidly progress. At this point, there was extensive involvement of her right neck and axilla, base of skull, mediastinum, and lungs (Fig. 1a). Treatment was changed to cemiplimab, a recombinant human IgG4 monoclonal antibody that blocks the PD-1 receptor. A near-complete response was seen clinically and per imaging after 4 months (Fig. 1b).

A year and a half after her initial cSCC diagnosis and 4 months under cemiplimab treatment, the patient presented to the ophthalmology clinic with bilateral vision deterioration lasting several months. Her best-corrected visual acuity was $20 / 30$ in both eyes. Clinical examination revealed a normal intraocular pressure, normal anterior segment, and mild nuclear sclerosis in both eyes. On funduscopic examination, hypopigmented lesions in a leopard pattern and pigmentary clumps were present in both eyes (Fig. 2a). Fundus autofluorescence imaging showed multiple scattered hypoautofluorescent lesions interspersed within areas of hyperautofluorescence in a symmetric pattern in both eyes in the macula and temporal to the macula (Fig. 2b). Spectral-domain enhanced-depth imaging optical coherence tomography revealed choroidal folds, pronounced and diffuse abnormalities at the level of the RPE, with alternating hyperreflective and irregular thickening, subretinal fluid, and scattered hyperreflective spots in the retina and choroid (Fig. 2c). The patient was diagnosed with BDUMP and referred to therapeutic plasma exchange (therapeutic plasmapheresis) with albumin, while 


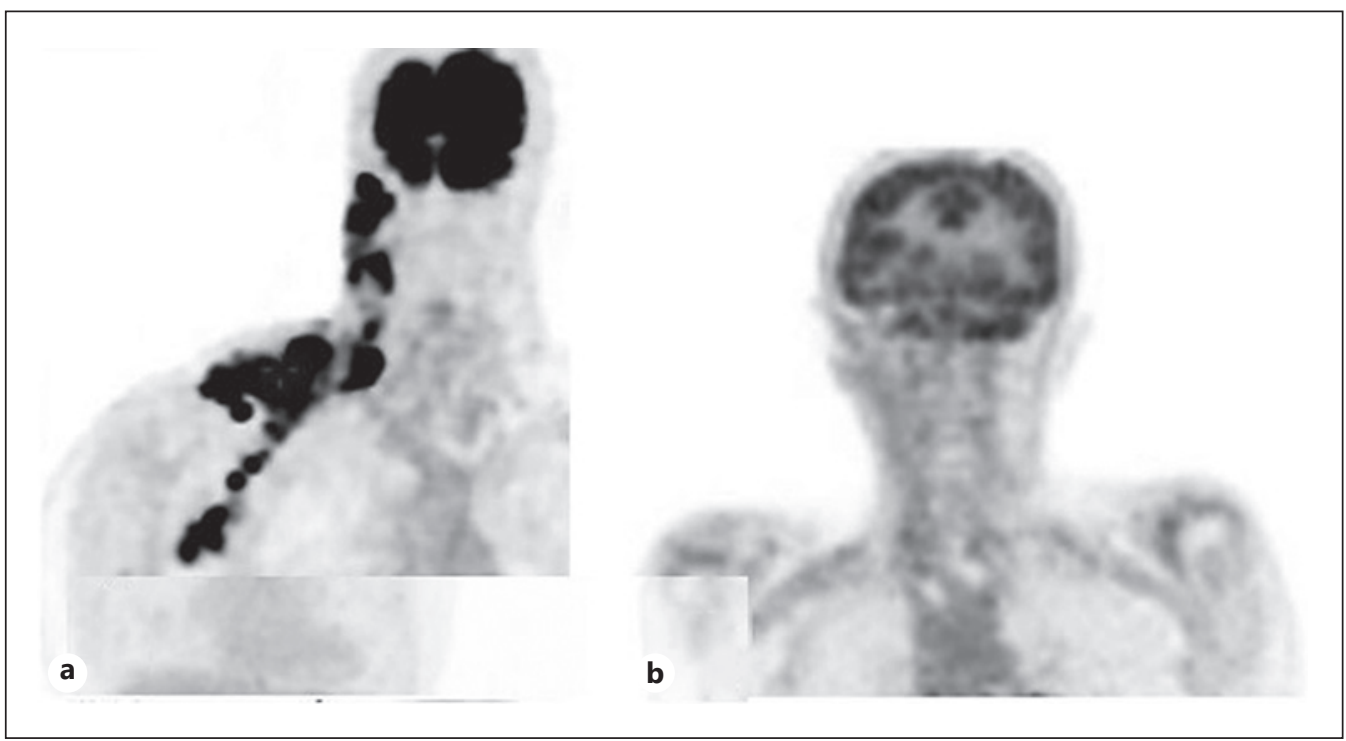

Fig. 1. a Head and neck PET-CT demonstrating metastatic SCC of the scalp. b Disease remission 4 months after cemiplimab. SCC, squamous cell carcinoma.

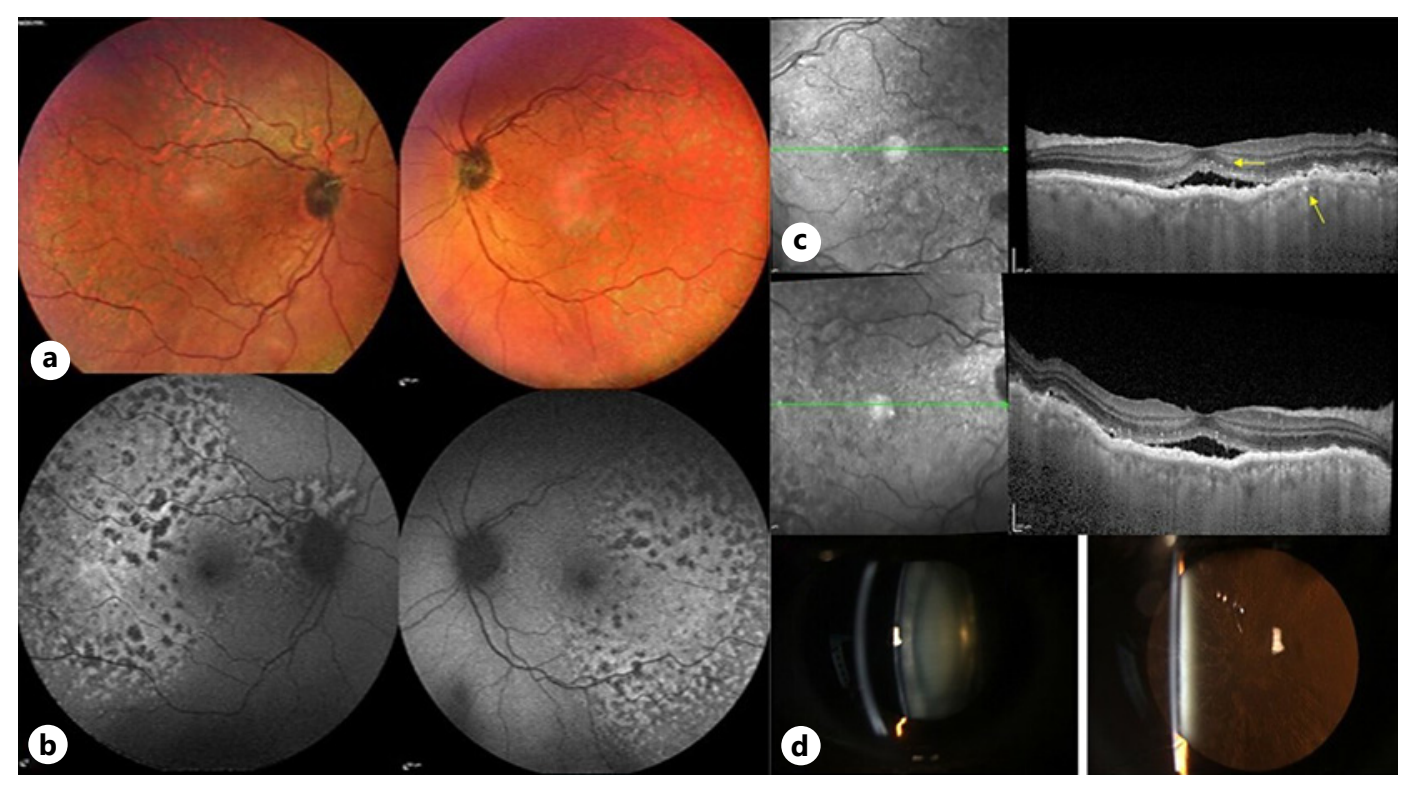

Fig. 2. a Funduscopic multicolor picture showing irregular, pigmented lesions with widespread multifocal patches of well-circumscribed areas of RPE hypopigmentation in a leopard pattern; FAF imaging showing multiple scattered hypoautofluorescent lesions interspersed within areas of hyperautofluorescence (b); SD-OCT showing choroidal folds, irregularity of the RPE, with RPE elevation and thickening, subretinal fluid, and scattered hyperreflective spots in the retina and choroid (c); slit lamp image of the RE showing advanced nuclear and posterior subcapsular cataract $(\mathbf{d})$.

treatment with cemiplimab was continued. After 3 cycles, complete resolution of the SRF was noticed. Overall, she underwent 9 cycles of therapeutic plasmapheresis. During follow-up, the cataract progressed and VA decreased to 20/60 in the right eye and 20/40 in the left eye (Fig. 2d), and the patient underwent cataract surgery. At the last follow-up, 12 months after 


\section{Case Reports in Ophthalmology}
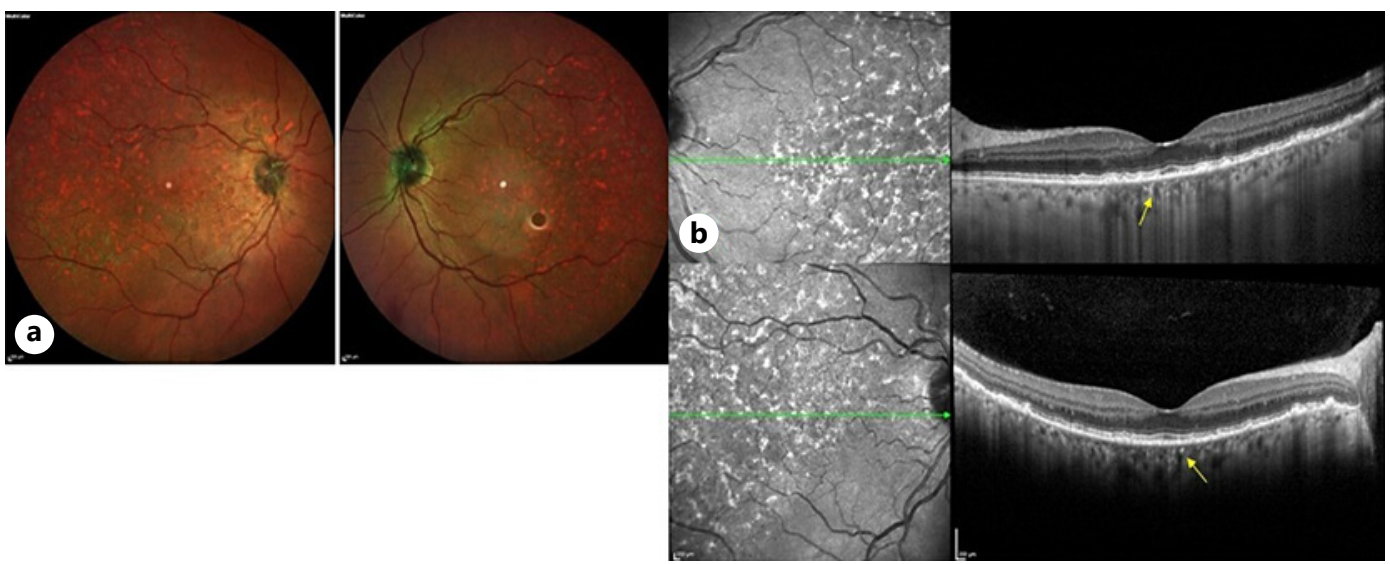

Fig. 3. a Funduscopic multicolor picture, 9 months after plasmapheresis treatment, showing the hypopigmented lesions in a leopard pattern. b SD EDI OCT 9 months after final plasmapheresis treatment showing resolution of choroidal folds, flattening and only mild irregularity of the RPE with absorption of all subretinal fluid. Note less hyperreflective spots in the retina and choroid.

completing the plasmapheresis treatment, best-corrected visual acuity improved to 20/20 in both eyes, there was no subretinal fluid, the choroidal folds resolved, and the RPE flattened and appeared less irregular (Fig. 3). The patient continued treatment with cemiplimab for 15 months with full regression of her primary disease (Fig. 1b). At the last follow-up, 3 months after cemiplimab cessation, there was no systemic disease recurrence.

\section{Discussion}

We describe the first case of BDUMP secondary to $\mathrm{CSCC}$, in a patient treated with cemiplimab (an anti-PD-1 therapy) for her metastatic cSCC. The patient, a 61-year-old female, fits the epidemiological profile of BDUMP patients described in the literature (female $60 \%$ and age 50-80) [1]. The patient presented with all 5 characteristic signs of the disease described by Gass et al. [3], including multiple subretinal round patches in the RPE, multiple elevated pigmented and nonpigmented uveal melanocytic lesions with early fluorescence of these lesions on FA, diffuse choroidal thickening, subretinal fluid, and rapid cataract development. BDUMP is a challenging diagnosis especially in patients treated with anti-PD-1 therapies (cemiplimab) as these drugs may cause ocular side effects including serous retinal detachment and Vogt-Koyanagi-Haradalike syndrome [5]. It is crucial to distinguish BDUMP from ocular manifestations secondary to the use of immunotherapy as it is of utmost importance to continue systemic immunotherapy treatment of the underlying systemic disease (Table 1). The continuation of immunotherapy treatment whilst on plasmapheresis enables control of the systemic as well as the ocular disease. Treatment with plasmapheresis is based on the rationale of removing certain proteins such as circulating antibodies from the bloodstream. Cultured melanocyte elongation and proliferation factor is an IgG fraction of circulating antibodies found in the serum of patients with BDUMP. This protein has been found to cause in vitro melanocytic proliferation [6]. Different plasmapheresis protocols have been described ranging from 3 treatments per week for 1 week to 3 treatments per week for 7 months $[7,8]$. The treatment was shown to be highly effective in maintaining favorable ocular disease outcome [7-12]. Our patient underwent overall 9 treatments during a 1-month period. After 1 month, all the subretinal fluid was absorbed, and we began to see an improvement in the RPE structure. In addition, she underwent a successful 
Table 1. Main differentiating features between BDUMP and PD-1 immunotherapy-related effects, specifically VKH-like effect of cemiplimab

\begin{tabular}{|c|c|c|}
\hline & BDUMP & $\begin{array}{l}\text { Anti-PD-1 immunotherapy-related } \\
\text { effects (VKH-like) }\end{array}$ \\
\hline Lens & $\begin{array}{l}\text { Rapidly progressive nuclear and posterior } \\
\text { subcapsular cataract }\end{array}$ & None described \\
\hline Fundus & $\begin{array}{l}\text { Irregular, pigmented lesions with widespread } \\
\text { multifocal patches of well-circumscribed areas } \\
\text { of RPE hypopigmentation in a leopard pattern }\end{array}$ & $\begin{array}{l}\text { Retinal vasculitis (occlusive or not), with } \\
\text { its consequent exudation, hemorrhage, or } \\
\text { macular edema; "sunset glow" fundus }\end{array}$ \\
\hline FAF & $\begin{array}{l}\text { Multiple scattered hypoautofluorescent lesions } \\
\text { interspersed within areas of hyperautofluorescence } \\
\text { in a leopard pattern compatible with the fundus } \\
\text { findings }\end{array}$ & $\begin{array}{l}\text { Larger areas of hyperautofluorescence } \\
\text { and hypoautofluorescence suggestive of } \\
\text { multifocal areas of regressed exudative } \\
\text { retinal detachment }\end{array}$ \\
\hline OCT & $\begin{array}{l}\text { Choroidal folds, irregularity of the RPE, with RPE } \\
\text { elevation and thickening, subretinal fluid, and } \\
\text { scattered hyperreflective spots in the retina and } \\
\text { choroid }\end{array}$ & $\begin{array}{l}\text { Choroidal folds, subretinal fluid with } \\
\text { multiple septae }\end{array}$ \\
\hline FA & $\begin{array}{l}\text { Reticular pattern of hypofluorescence surrounded } \\
\text { by a background of choroidal hyperfluorescence } \\
\text { creating a characteristic mosaic with } \\
\text { hyperfluorescent patches }\end{array}$ & $\begin{array}{l}\text { Early hypofluorescence and late } \\
\text { hyperfluorescence in the areas of retinal } \\
\text { pigment epithelial changes, consistent } \\
\text { with multifocal exudative retinal } \\
\text { detachments }\end{array}$ \\
\hline
\end{tabular}

BDUMP, bilateral diffuse uveal melanocytic proliferation; anti-PD-1, anti-programmed cell death protein 1.

cataract surgery in both eyes with visual acuity improvement. Our case is the first case of BDUMP related to SCC of the scalp and has the longest follow-up as compared to previously published BDUMP cases. Twelve months after last plasmapheresis treatment, the patient demonstrated a favorable ocular result; VA was excellent in both eyes, there was no recurrence of subretinal fluid, no evidence of choroidal folds, and RPE appearance improved. Thanks to the quick diagnosis of BDUMP, an appropriate treatment with plasmapheresis was given while maintaining systemic treatment with cemiplimab that achieved complete response of her metastatic cSCC. Theoretically, the filtration process of plasmapheresis could lower the desired antibody levels of the cemiplimab, and this phenomenon was not described in the literature. However, due to the short-term treatment schedule of plasmapheresis ( 1 month) and the devastating visual loss, we planned for the patient to receive cemiplimab during and after the last plasmapheresis treatment. The systemic effect of cemiplimab was not compromised in this case, as seen on the last oncologic follow-up and PET-CT.

\section{Statement of Ethics}

The subject has given her written informed consent to publish the case (including publication of images); this study has been granted an exemption from requiring ethical approval by the Institutional Review Board (IRB) of the Tel Aviv Medical Center, Israel.

\section{Conflict of Interest Statement}

The authors have no conflicts of interest to declare.

\section{Karger's}




\author{
Funding Sources
}

This manuscript did not receive any funding.

\title{
Author Contributions
}

Etti Katzburg conceived and designed, collected the data, and wrote the manuscript. Dinah Zur conceived and designed, contributed data, and revised the manuscript. Orit Gutfeld contributed data and revised the manuscript. Ilya Kirshner contributed data and revised the manuscript. Zohar Habot-Wilner conceived and designed, collected the data, and revised the manuscript.

\section{Data Availability Statement}

All data generated or analyzed during this study are included in this article. Further enquiries can be directed to the corresponding author.

\section{References}

1 Klemp K, Kiilgaard JF, Heegaard S, Nørgaard T, Andersen MK, Prause JU. Bilateral diffuse uveal melanocytic proliferation: case report and literature review. Acta Ophthalmol. 2017;95(5):439-45.

2 Mittal R, Cherepanoff S, Thornton S, Kalirai H, Damato B, Coupland SE. Bilateral diffuse uveal melanocytic proliferation: molecular genetic analysis of a case and review of the literature. Ocul Oncol Pathol. 2016;2(2):94-9.

3 Gass JD, Gieser RG, Wilkinson CP, Beahm DE, Pautler SE. Bilateral diffuse uveal melanocytic proliferation in patients with occult carcinoma. Arch Ophthalmol. 1990;108(4):527-33.

4 Miles SL, Niles RM, Pittock S, Vile R, Davies J, Winters JL, et al. A factor found in the igg fraction of serum of patients with paraneoplastic bilateral diffuse uveal melanocytic proliferation causes proliferation of cultured human melanocytes. Retina. 2012;32(9):1959-66.

5 Libtayo (cemiplimab).

6 Mets RB, Golchet P, Adamus G, Anitori R, Wilson D, Shaw J, et al. Bilateral diffuse uveal melanocytic proliferation with a positive ophthalmoscopic and visual response to plasmapheresis. Arch Ophthalmol. 2011;129(9):1235-8.

7 Miles SL, Niles RM, Pittock S, Vile R, Davies J, Winters JL, et al. A factor found in the igg fraction of serum of patients with paraneoplastic bilateral diffuse uveal melanocytic proliferation causes proliferation of cultured human melanocytes. Retina. 2012;32(9):1959-66.

8 Jansen JC, Van Calster J, Pulido JS, Miles SL, Vile RG, Van Bergen T, et al. Early diagnosis and successful treatment of paraneoplastic melanocytic proliferation. Br J Ophthalmol. 2015;99(7):943-8.

9 Navajas EV, Simpson ER, Krema H, Hammoudi DS, Weisbrod D, Bernardini M, et al. Cancer-associated nummular loss of RPE: expanding the clinical spectrum of bilateral diffuse uveal melanocytic proliferation. Ophthalmic Surg Lasers Imaging. 2011;42:e103-6.

10 Jaben EA, Pulido JS, Pittock S, Markovic S, Winters JL. The potential role of plasma exchange as a treatment for bilateral diffuse uveal melanocytic proliferation: a report of two cases. J Clin Apher. 2011;26(6):356-61.

11 Thompson AC, Chen X, Mruthyunjaya P. A case of progressive dimming vision. JAMA Ophthalmol. 2018; 136(4):434-5.

12 Höh AE, Holz FG, Dithmar S. [Bilateral diffuse uveal melanocytic proliferation in a patient with metastatic breast cancer]. Ophthalmologe. 2014;111(10):961-4.

\section{Karger'₹}

